\title{
Enhanced recovery in the management of mild gallstone pancreatitis: a prospective cohort study
}

\author{
Xin Zhao $\cdot$ Da-Zhi Chen $\cdot$ Ren Lang • \\ Zhong-Kui Jin · Hua Fan · Tian-Ming Wu • \\ Xian-Liang $\mathrm{Li} \cdot$ Qiang He
}

Received: 28 November 2011/ Accepted: 30 March 2012/Published online: 9 October 2012

(C) The Author(s) 2012. This article is published with open access at Springerlink.com

\begin{abstract}
Purpose The aim of this study was to establish enhanced recovery protocols for the management of mild gallstone pancreatitis.

Methods Sixty consecutive patients were divided into enhanced recovery and traditional recovery (TR) groups in a randomized observational study. The basic enhanced recovery elements included early laparoscopic cholecystectomy, restrictive endoscopic intervention, and early oral nutrition. The incidence of complications, readmission, length of stay, and total medical cost were analyzed during the hospital course.

Results The length of hospital stay and medical cost were significantly lower in the enhanced recovery group in comparison to the TR group: 5.9 days vs. 10.6 days $(P<0.01)$ and $¥ 10,023$ vs. $¥ 15,035 \quad(P<0.01)$. The complications and readmission rates in the two groups were similar.

Conclusions The implementation of enhanced recovery protocols is feasible in the management of mild gallstone pancreatitis. The utilization of these protocols can achieve shorter hospital stays and reduced costs, with no increase in either the re-admission or peri-operative complication rates.
\end{abstract}

Keywords Enhanced recovery - Acute gallstone pancreatitis · Laparoscopic cholecystectomy · Endoscopic retrograde cholangiopancreatography

X. Zhao - D.-Z. Chen $(\varangle) \cdot$ R. Lang · Z.-K. Jin · H. Fan · T.-M. Wu X.-L. Li $\cdot$ Q. He

Department of Hepatobiliary and Pancreatospleenic Surgery,

Beijing Chaoyang Hospital Affiliated to Capital Medical

University, Beijing 100020, China

e-mail: chendazhi@medmail.com.cn

\author{
Abbreviations \\ ABP Acute biliary pancreatitis \\ AGP Acute gallstone pancreatitis \\ ALT Alanine aminotransferase \\ CBD Common bile duct \\ CRP C-reactive protein \\ ER Enhanced recovery \\ ERCP Endoscopic retrograde cholangiopancreatography \\ LC Laparoscopic cholecystectomy \\ TR Traditional recovery
}

\section{Introduction}

Enhanced recovery (ER) pathways in surgical practices have recently reduced the duration of hospital stays and have been applied successfully in major surgery. They have been found to pose no risk to patients in terms of morbidity and mortality in colorectal [1], breast, and even pancreatic surgery [2, 3]. Although ER pathways could make an important contribution to distressed health care systems, they are used in less than one-third of surgical practices in the US and the UK [4, 5]. Acute pancreatitis may lead to a systemic inflammatory response syndrome with significant morbidity and mortality in $20 \%$ of patients, thus ER practices are limited citing safety concerns.

"Gallstones" are the most common etiology of acute pancreatitis [6, 7]. Biliopancreatic obstruction is usually transient during the course of this disease because the offending stone passes rapidly into the duodenum; thus, acute pancreatitis is associated with minimal organ dysfunction and an uneventful recovery [8]. Therefore, the establishment of ER pathways could greatly reduce the time required for full recovery and could be applied to the 
management of mild acute gallstone pancreatitis (AGP). No previous randomized trial has focused on the adoption of ER principles such as the optimal time for cholecystectomy, use of enteral nutrition, and the choice of early endoscopic intervention in this subgroup of pancreatitis.

This study sought to establish protocols based on ER pathways for the treatment of mild AGP and to determine its safety, efficacy, and economic implications in a prospective cohort trail.

\section{Patients and methods}

All patients who presented to the emergency ward within $24 \mathrm{~h}$ after the onset of acute pancreatitis from June 2009 to October 2011 were evaluated for study eligibility. The diagnosis of AGP was based on the presence of three criteria: (1) acute upper abdominal pain; (2) serum amylase more than three times the upper limit of normal combined with an increase in alanine aminotransferase (ALT) $>150 \mu / 1$ [9]; and (3) cholelithiasis and the characteristic findings of acute pancreatitis on admission CT scan or ultrasound. A diagnosis of AGP was established when 2 of 3 items were positive [10]. Patients with an APACHE II score $<8$ together with Balthazar CT score $<4$ were considered to have mild AGP. The exclusion criteria were an age below 18 years, pregnancy, patients with acute cholangitis, or with serious comorbid conditions that precluded laparoscopic cholecystectomy (LC).

Patients were randomized to receive either an ER pathway or traditional recovery (TR) care, using sealed envelopes. The envelopes were randomized in blocks and opened by a surgeon not otherwise engaged in the study. Randomization was performed once CT examination and laboratory studies had been completed, and the severity of pancreatitis was evaluated.

Supportive care in both groups included controlled fluid resuscitation, supplemental oxygen administered during the first $24-48 \mathrm{~h}$ and analgesia. Intravenous prophylactic antibiotics were administered to avoid the edema of duodenal papilla caused by acute inflammation during the early stage of mild AGP. Antibiotics were discontinued 5 days after hospitalization in the absence of the risk of pancreatic infection. No patient underwent gastrointestinal decompression or urinary catheterization before surgery. LC was performed during the initial hospital admission.

The TR group usually initiated early oral intake of limited calories when abdominal pain had subsided such that abdominal tenderness had markedly decreased, nausea and vomiting had ceased, and bowel sounds were present. The early use of endoscopic retrograde cholangiopancreatography (ERCP) was not restricted, in patients with jaundice, or known or suspected choledocholithiasis.
The contents of ER pathways mainly included early LC routinely on the second day after admission, restrictive ERCP before surgery, and early oral nutritional support. A special study surgeon provided the patients and their relatives with detailed information about their recovery program on the first day of hospitalization, to help build confidence that their postoperative pain would be reduced. Patients in the ER group underwent LC 2 days after admission. The restrictive indication of endoscopic intervention was the confirmation of common bile duct (CBD) stones combined with aggravated jaundice. The patients were allowed the early oral intake of carbohydrates from the day of admission. Table 1 compares the principles of management between the ER group and the TR group.

The discharge criteria were the same for the two groups: tolerance to solid diet; relief of abdominal pain; no surgical complications; and normal direct bilirubin level.

The primary endpoint was the intention to treat. Dichotomous variables were recorded as the absolute frequencies (number of cases) and relative frequencies (percentages). Continuous variables were recorded as the means and standard deviations (SD) or the median plus maximal and minimal values. Continuous variables were compared using two-sample $t$ test when the data were normally distributed. The Wilcoxon rank sum test was used in the absence of normal distribution. Categorical data were analyzed using the Chi-square or Fisher exact test as appropriate. $P<0.05$ was considered to be significant. All analyses were conducted using SPSS version 13 software package.

\section{Results}

Of the 202 available gallstone pancreatitis patients, 60 were eligible for inclusion. No deaths occurred during the observation period in either group. The ER and TR groups were comparable in terms of age, gender, and severity of AGP (Table 2).

There were no significant differences between the two groups in the levels of ALT, direct bilirubin, and C-reactive protein (CRP) on admission. All patients underwent cholecystectomy during the initial hospitalization. There was no significant differences between the ER and TR groups in the incidence of conversion of LC to an open procedure $(P=0.61)$. The exploration of the CBD was performed in one $(3.3 \%)$ of 30 patients in the ER group and five $(16.7 \%)$ of 30 patients in the TR group. Only one patient in the ER group underwent preoperative ERCP under the restrictive criteria. Endoscopic intervention was used in 11 patients in the TR group and detected that CBD stones in seven cases. 
Table 1 Principles of enhanced recovery pathway and traditional care

\begin{tabular}{|c|c|c|}
\hline & ER pathway & TR care \\
\hline \multirow[t]{4}{*}{ Preoperative support } & $\begin{array}{l}\text { Patients were given information on mild AGP and the } \\
\text { ER pathway }\end{array}$ & Patients were informed in the standard manner \\
\hline & $\begin{array}{l}\text { Early oral intake of carbohydrates from the day of } \\
\text { admission }\end{array}$ & $\begin{array}{l}\text { Limited calories, usually initiated when abdominal pain had } \\
\text { subsided }\end{array}$ \\
\hline & Restrictive early endoscopic intervention & $\begin{array}{l}\text { Early ERCP was not restricted, in patients with jaundice, or } \\
\text { known or suspected CBD stones }\end{array}$ \\
\hline & Provision of $10 \%$ glucose (total $400 \mathrm{ml}$ ) $4 \mathrm{~h}$ before LC & No meals or water during the $8 \mathrm{~h}$ before surgery \\
\hline Day of operation & $\begin{array}{l}\text { Exploration of CBD, intra-operative cholangiography, } \\
\text { or abdominal drainage was omitted } \\
\text { Liquid diet within } 6 \mathrm{~h} \text { after LC if no vomiting or nausea } \\
\text { Patients encouraged to mobilize }\end{array}$ & $\begin{array}{l}\text { Exploration of CBD confirmed presence of stones before } \\
\text { surgery, routine placement of abdominal drainage } \\
\text { Liquid diet } 6 \mathrm{~h} \text { after operation } \\
\text { No mobilization }\end{array}$ \\
\hline \multicolumn{3}{|l|}{ Postoperative care } \\
\hline Day 1 after surgery & $\begin{array}{l}\text { Oral intake of normal diet } \\
\text { Expand mobilization }\end{array}$ & $\begin{array}{l}\text { Low fat diet } \\
\text { Start mobilization }\end{array}$ \\
\hline Day 2 after surgery & Evaluate discharged criteria & $\begin{array}{l}\text { Oral intake of normal diet, expand mobilization, and assess } \\
\text { discharge criteria }\end{array}$ \\
\hline
\end{tabular}

$E R$ enhanced recovery, $T R$ traditional recovery, $A G P$ acute gallstone pancreatitis, $E R C P$ endoscopic retrograde cholangiopancreatography, $C B D$ common bile duct, $L C$ laparoscopic cholecystectomy

Table 2 Overview of the patient characteristics and surgery

\begin{tabular}{|c|c|c|c|}
\hline & $\begin{array}{l}\mathrm{ER} \\
(n=30)\end{array}$ & $\begin{array}{l}\text { TR } \\
(n=30)\end{array}$ & $P$ value \\
\hline Age (years) & $\begin{array}{l}56 \\
(35-81)\end{array}$ & $\begin{array}{l}58 \\
\quad(28-79)\end{array}$ & 0.30 \\
\hline Gender ratio: male/female & $13 / 17$ & $12 / 18$ & 0.79 \\
\hline APACHE II score & 3.2 & 3.3 & 0.44 \\
\hline CT severity index & 0.9 & 1.1 & 0.14 \\
\hline $\operatorname{ALT}(\mu \mathrm{l})$ & 225.1 & 198.1 & 0.13 \\
\hline DBil $(\mu \mathrm{mol} / 1)$ & 35.8 & 37.4 & 0.38 \\
\hline $\mathrm{CRP}(\mathrm{mg} / \mathrm{l})$ & 35.4 & 36.8 & 0.37 \\
\hline $\begin{array}{l}\text { Type of surgery: LC/LC } \\
\text { converting to open }\end{array}$ & $29 / 1$ & $27 / 3$ & 0.61 \\
\hline
\end{tabular}

$E R$ enhanced recovery, $T R$ traditional recovery, $A L T$ alanine aminotransferase, $D B i l$ direct bilirubin, $C R P$ C-reactive protein, $L C$ laparoscopic cholecystectomy

Table 3 shows the medical complications, readmission rate, hospital stay, and total hospital cost. The rate of readmission was $6.67 \%$ in the ER group, in comparison to $3.33 \%$ in the TR group $(P=1.00)$. Some patients in both groups were readmitted for residual stones in the CBD. The most common complication was acute lung injury. The complication rate was similar in the two groups and no surgical complications occurred. There was a significant difference between the two groups in the mean duration of hospital stay $(P<0.01)$, mainly due to the number of preoperative days $(2.4$ vs. 6.8 days, $P<0.01)$. Most patients in the two groups recovered rapidly after surgery and were discharged within 4 days $(P=0.30)$. The total hospital cost in the ER group was significantly less than
Table 3 Complications, cost and duration of hospital stay

\begin{tabular}{|c|c|c|c|}
\hline & $\begin{array}{l}\mathrm{ER} \\
(n=30)\end{array}$ & $\begin{array}{l}\mathrm{TR} \\
(n=30)\end{array}$ & $P$ value \\
\hline Complications & 1 & 2 & 1.00 \\
\hline Readmission & 2 & 1 & 1.00 \\
\hline $\begin{array}{l}\text { Duration of hospital stay } \\
\text { (day) }\end{array}$ & 5.9 & 10.6 & $<0.01$ \\
\hline Days before operation (day) & 2.4 & 6.8 & $<0.01$ \\
\hline Days after operation (day) & 3.7 & 3.5 & 0.30 \\
\hline Total hospital cost (¥) & 10023 & 15035 & $<0.01$ \\
\hline
\end{tabular}

$E R$ enhanced recovery, $T R$ traditional recovery

that in the TR group $(P<0.01)$ due to the shorter hospital stay, fewer medical treatments were required during the preoperative period and less use of ERCP.

\section{Discussion}

Enhanced recovery pathways involve various techniques for the care of patients undergoing elective surgery. The methods used include minimally invasive procedures, optimal pain control, and aggressive postoperative rehabilitation, including early oral nutrition and ambulation. The present study indicated that a policy of ER in patients with mild AGP can result in a significantly reduced length of hospital stay and total cost with no increase in complications, readmission rate, or mortality.

The shorter hospital stay in the current series was mainly due to early LC, restrictive endoscopic intervention, and oral nutritional support initiated as early as possible. 
Recurrent episodes of acute pancreatitis occur in as many as $30-50 \%$ of patients with gallstone pancreatitis during the waiting period before interval LC. The guidelines recommend that all patients with biliary pancreatitis should undergo definitive management of gallstones during the same hospital admission, unless a clear plan has been made for definitive treatment within the next 2 weeks [5]. However, the timing of cholecystectomy in AGP remains controversial. The mean length of the hospital stay was reduced by 5 days in the ER group in comparison to the TR group, due largely to a reduction in the preoperative waiting time. David et al. concluded that a policy of early cholecystectomy in patients with mild-to-moderate gallstone pancreatitis resulted in a significantly reduced length of hospital stay with no increase in complications or mortality [11]. Rajeev supports the concept that early LC should be preferred in all patients with mild acute biliary pancreatitis (ABP) because it protects against further attacks of ABP during the wait for interval cholecystectomy [12]. In addition, the rate of conversion from laparoscopic to open cholecystectomy is greater in interval LC than in early LC. The current study found that the rate of conversion to an open procedure was less in the ER group than in the TR group, although the difference was not statistically significant. Routine intraoperative cholangiography was omitted in the ER group, because the incidence of bilo-pancreatic complications in patients with mild gallstone pancreatitis who undergo cholecystectomy is very low [13].

Endoscopic retrograde cholangiopancreatography is a challenging endoscopic procedure, with a reported rate of procedure-related complications of approximately 5-10\% $[14,15]$. The need for early endoscopic intervention is controversial regarding patients with AGP [16]. Charles et al. believes that all cases of suspected gallstones pancreatitis with known or suspected choledocholithiasis should undergo systematic biliary sphincterotomy [17]. In contrast, Alejandro et al. reported that early endoscopic intervention failed to reduce systemic and local inflammation in patients with AGP and biliopancreatic obstruction [18]. Early endoscopic intervention is not required if acute cholangitis can be safely excluded and should not be considered a standard indication. Recent studies have demonstrated a $71-88 \%$ rate of spontaneous disobstruction within $48 \mathrm{~h}$ after the onset of ABP (and a subsequent uneventful course of acute pancreatitis) [19, 20]. Indeed, only a small subgroup of patients might have a theoretical justification for ERCP. Several clinical studies have shown that the duration of obstruction $(>48 \mathrm{~h})$ is a critical determinant for ERCP [21]. The current series demonstrated that the levels of direct bilirubin decreased to normal spontaneously without emergent endoscopic intervention in $98 \%$ (29/30) of patients in the ER group. Early ERCP should be limited in mild AGP. The indication for endoscopic intervention should be confirmed CBD stones combined with aggravated jaundice. The length of hospital stay can be shortened by avoiding the risks of early endoscopic intervention.

"Pancreatic rest" or reduction of exocrine secretion is very important in the treatment of patients with acute pancreatitis. Enteral nutrition has no positive impact on the course of mild acute pancreatitis and it is only recommended in patients who cannot consume normal food after 5-7 days [22]; as a consequence, several days of fasting is widely employed in most of these patients. However, the concept of pancreatic rest remains theoretical and has been inadequately tested; there are no randomized studies demonstrating that this practice hastens recovery in acute pancreatitis. Of the three main groups of nutrients (proteins, lipids, and carbohydrates), carbohydrates have the weakest stimulatory effect on pancreatic secretion [23]. The current study initiated the oral intake of carbohydrates on the day of admission, once abdominal pain had subsided, to shorten the fasting time in the ER group. No relapses of pain occurred within $48 \mathrm{~h}$ of the start of oral feeding. This change in approach in comparison to TR care may be implemented in AGP to shorten the preoperative period.

\section{Conclusions}

The present study indicates that ER pathways can be implemented in the management of mild gallstone pancreatitis. Such protocols can lead to shorter hospital stays and reduced cost, with no increase in either the re-admission or peri-operative complication rates.

Conflict of interest Xin Zhao, Da-Zhi Chen, Ren Lang, Zhong-Kui Jin, Hua Fan, Tian-Ming Wu, Xian-Liang Li and Qiang He have no conflicts of interest.

Open Access This article is distributed under the terms of the Creative Commons Attribution License which permits any use, distribution, and reproduction in any medium, provided the original author(s) and the source are credited.

\section{References}

1. Ramirez JM, Blasco JA, Rig JV, Maeso-Martinez S, Casal JE, Esteban F, et al. Enhanced recovery in colorectal surgery: a multicentre study. BMC Surg. 2011;11:9.

2. Arsalani-Zadeh R, ElFadl D, Yassin N, MacFie J. Evidencebased review of enhancing postoperative recovery after breast surgery. Br J Surg. 2011;98:181-96.

3. Ypsilantis E, Praseedom RK. Current status of fast-track recovery pathways in pancreatic surgery. JOP $\mathrm{J}$ Pancreas (Online). 2009;10(6):646-50. 
4. UK Working Party on Acute Pancreatitis. UK guidelines for the management of acute pancreatitis. Gut. 2005;54:1-9.

5. Banks PA, Freeman ML. Practice guidelines in acute pancreatitis. Am J Gastroenterol. 2006;101:2379-400.

6. Frossard JL, Steer ML, Pastor CM. Acute pancreatitis. Lancet. 2008;371:143-52.

7. Spanier BW, Dijkgraaf MG, Bruno MJ. Epidemiology, aetiology and outcome of acute and chronic pancreatitis: an update. Best Pract Res Clin Gastroenterol. 2008;22:45-63.

8. Neoptolemos JP. The theory of 'persisting' common bile duct stones in severe gallstone pancreatitis. Ann R Coll Surg Engl. 1989;71:326-31.

9. Levy P, Boruchowicz A, Hastier P, Pariente A, Thevenot T, Frossard JL, et al. Diagnostic criteria in predicting a biliary origin of acute pancreatitis in the era of endoscopic ultrasound: multicentre prospective evaluation of 213 patients. Pancreatology. 2005;5:450-6.

10. Kimura Y, Arata S, Tadahiro T, Hirata K, Yoshida M, Mayumi T, et al. Gallstone-induced acute pancreatitis. J Hepatobiliary Pancreat Sci. 2010;17:60-9.

11. Rosing DK, de Virgilio C, Yaghoubian A, Putnam BA, El MM, Kaji A, et al. Early cholecystectomy for mild to moderate gallstone pancreatitis shortens hospital stay. J Am Coll Surg. 2007;205(6):762-6.

12. Sinha R. Early laparoscopic cholecystectomy in acute biliary pancreatitis: the optimal choice? HPB. 2008;10:332-5.

13. Horwood J, Akbar F, Davis K, Morgan R. Prospective evaluation of a selective approach to cholangiography for suspected common bile duct stones. Ann R Coll Surg Engl. 2010;92:206-10.

14. Freeman ML, Nelson DB, Sherman S, Haber GB, Herman ME, Dorsher PJ, et al. Complications of endoscopic biliary sphincterotomy. N Engl J Med. 1996;335:909-18.
15. Loperfido S, Angelini G, Benedetti G, Chilovi F, Costan F, De Berardinis F, et al. Major early complications from diagnostic and therapeutic ERCP: a prospective multicenter study. Gastrointest Endosc. 1998;48:1-10.

16. Vitale GC. Early management of acute gallstone pancreatitis. Ann Surg. 2007;245:18-9.

17. Shrode CW, Kahaleh M. Early ERCP in acute gallstone pancreatitis without cholangitis: a need for systematic biliary sphincterotomy! JOP. J Pancreas (Online). 2009;10(6):701-2. author reply 703-4.

18. Oria A, Cimmino D, Ocampo C, Silva W, Kohan G, Zandalazini $\mathrm{H}$, et al. Early endoscopic intervention versus early conservative management in patients with acute gallstone pancreatitis and biliopancreatic obstruction a randomized clinical trial. Ann Surg. 2007;245:10-7.

19. Acosta JM, Rubio GOM, Rossi R, Chinellato AV, Pellegrini CA. Effect of duration of ampullary gallstone obstruction on severity of lesions of acute pancreatitis. J Am Coll Surg. 1997;184: 499-505.

20. Acosta JM, Pellegrini CA, Skinner DB. Etiology and pathogenesis of acute biliary pancreatitis. Surgery. 1980;88:118-25.

21. Acosta JM, Katkhouda N, Debian KA, Groshen SG, Tsao-Wei DD, Berne TV. Early ductal decompression versus conservative management for gallstone pancreatitis with ampullary obstruction: a prospective randomized clinical trial. Ann Surg. 2006;243: $33-40$.

22. Ioannidis O, Lavrentieva A, Botsios D. Nutrition support in acute pancreatitis. JOP. J Pancreas (Online). 2008;9(4):375-90.

23. Meier R, Ockengab J, Pertkiewicz M, Pap A, Milinic N, MacFie $\mathrm{J}$, et al. ESPEN guidelines on enteral nutrition: pancreas. Clin Nutr. 2006;25:275-84. 